\title{
The Internet Development Process: Observations and Reflections
}

\author{
Pål Spilling \\ University Graduate Center (UNIK), Kjeller, Norway \\ paal@unik.no
}

\begin{abstract}
Based on the experience of being part of the team that developed the internet, the author will look back and provide a history of the Norwegian participation. The author will attempt to answer a number of questions such as why was The Norwegian Defense Research Establishment (FFI) invited to participate in the development process, what did Norway contribute to in the project, and what did Norway benefit from its participation?
\end{abstract}

Keywords: ARPANET, DARPA, Ethernet, Internet, PRNET.

\section{A Short Historical Résumé}

The development of the internet went through two main phases. The first one laid the foundation for packet switching in a single network called ARPANET. The main idea behind the development of ARPANET was resource sharing [1]. At that time computers, software, and communication lines were very expensive, meaning that these resources had to be shared among as many users as possible. The development started at the end of 1968. The U.S. Defense Advanced Research Project Agency (DARPA) funded and directed it in a national project. It became operational in 1970; in a few years, it spanned the U.S. from west to east with one arm westward to Hawaii and one arm eastward to Kjeller, Norway, and then onwards to London.

The next phase, the "internet project," started in the latter half of 1974 [2]. The purpose of the project was to develop technologies to interconnect networks based on different communication principles, enabling end-to-end communications between computers connected to different networks. It was organized as an international project financed and directed by DARPA with Bob Kahn and later professor Vint Cerf as project leader. The internet project had twenty to thirty participants from academia and research institutions in the U.S., four from University College London (UCL), and two from FFI in Norway. Professor Vint Cerf at Stanford University, with his team of PhD students, developed the initial specifications of the TCP. In addition, Dag Belsnes from the University of Oslo had leave of absence and stayed nine months in Cerf's group from April 1974. His task was to analyze the internet protocol (TCP/IP) to find possible weaknesses in it. Three sites - Stanford University, BBN (Bolt, Beranek and Newman) in Boston, and University College in London (UCL) were responsible for independent implementation of these specifications. Then, these independent implementations were thoroughly tested against one another. At the end 
of 1979 , the technology was mature enough to be proposed and subsequently accepted as a standard for the U.S. defense in the middle of 1980 .

At the end of 1972, DARPA initiated a mobile network development, called the Packet Radio Network (PRNET) [3]. It was inspired by the Aloha project in Hawaii, based on the use of radio-based links, for terminal access to a set of central computer facilities in Honolulu, from various islands in the Hawaiian archipelago. In addition, DARPA had a desire to develop a packet-based satellite network [4].

These activities may in part be considered as incentives for DARPA to interconnect networks based on different technologies. In addition, Bob Metcalf at XEROX-PARC developed the local area network, called Ethernet, in 1973. SRI International conducted the first true internet demonstration in November 1977, involving ARPANET, SATNET, and PRNET.

\section{Why was Norway Invited to Participate in the Internet Project?}

The Nuclear-Test-Ban-Treaty and the close vicinity to Russia made Norway an attractive location for a seismic detection facility, to detect underground atomic test explosions. The Research Council of Norway, with funding support from DARPA, established NORSAR (Norwegian Seismic Array) in 1970 at Kjeller. A 2.4 kb/s line was installed from NORSAR to the Seismic Data Analysis Center (SDAC) in Washington DC. The line went via cable to the British satellite ground station at Goonhilly, and then by satellite to the U.S. When the Nordic satellite ground station at Tanum Sweden became operational in 1971, the NORSAR-SDAC line was relocated to go via Tanum.

DARPA had initially wanted to cooperate with Donald Davies, a research scientist at the National Physics Laboratory (NPL) in the UK. Davies had developed a packetbased network, enabling distributed terminals to connect to a set of host computers [5]. Because England just had applied for membership in EU, this forced NPL to focus on European research issues. This made it impossible for DARPA to link its ARPANET to the network at NPL and cooperate with Davies.

DARPA then turned to Norway, inviting the research department of the Norwegian Telecommunications Administration (Telenor-R\&D) and FFI to participate in the development of satellite-based packet communications, believed to be of importance to the Norwegian shipping industry. In addition, the collaboration between DARPA, UCL, and FFI would make it more efficient and cheaper to link up both UCL and FFI to ARPANET, via the NORSAR - Tanum - SDAC line. Telenor showed little interest in participating, while the invitation was accepted by FFI. As a result, an ARPANET packet-switching node was installed at NORSAR in June of 1973. The ARPANET nodes, called Interface Message Processors (IMPs) were developed in two versions, one version provided attachment for four host computers, the other provided attachments for three hosts and sixty-three terminals, called IMP and TIP respectively. In September 1973, another node was installed at UCL and it was connected with a leased line to the node at NORSAR. FFI's participation in the internet project started in the beginning of 1975. Somewhat later, after several meetings between FFI and Telenor, Telenor was willing to provide, free of charge for the duration of the project, the uplink to the satellite and a $48 \mathrm{~kb} / \mathrm{s}$ line from Kjeller to the satellite ground station at Tanum, Sweden. 


\section{The Main Norwegian Contributions}

The internet project was actually the integration of several interconnected projects, and included the internet development project, the SATNET project, the packet radio project, and the packet speech project. In the period 1976 through 1979, FFI was heavily involved in the development of SATNET [6]. The purpose of the SATNET project was to explore the feasibility of operating a $64 \mathrm{~kb} / \mathrm{s}$ channel in the INTELSAT system, multiplexed between a set of ground stations in a packet switched modus. Three ground stations were involved in the project, one at Etam in West Virginia on the U.S. East Coast, one at Goonhilly at the English West Coast, and the third one at the Nordic satellite ground station in Tanum, Sweden. To enable the packet-switched operation of the satellite channel, packet-switching nodes called Satellite-IMPs (SIMPs) were installed in the ground stations - interfacing with the satellite channel equipment. Each SIMP was then interconnected, via a leased line, with a gateway computer connected to the ARPANET. The gateway, a PDP-11/45, connected to the Tanum-SIMP was located at FFI.

The SATNET research program, directed by Bob Kahn, was performed as a joint effort between Linkabit Corporation in San Diego, University of California in Los Angeles (UCLA), Bolt Beranek and Newman (BBN) in Boston, Communications Satellite Corporation (COMSAT) in Gaithersburg, Maryland, University College London (UCL), and FFI in Norway. Linkabit had the project's technical leadership. BBN was responsible for the development of the SIMPs, including the various channel-access algorithms the participants wanted to test. The project participants met about four times a year, with the meeting location circulating among the participating institutions.

Yngvar Lundh, with Pål Spilling as the main work force, headed the Norwegian contingent. Finn-Arve Aagesen, later a professor at NTNU in Trondheim, stayed with the project for twelve months. He was responsible for performing simulation studies of the most promising channel access algorithm, the "Contention-based, PriorityOriented Demand Access" algorithm (C-PODA). Pål Spilling developed management software on a FFI-based computer, to control artificial traffic generation in the SIMPs, and to fetch data collected in the SIMPs during measurements. Several access algorithms were studied experimentally, among others TDMA, Reservation-TDMA, and C-PODA [7]. Kleinrock's Network Measurements Group at UCLA also performed measurements and simulations.

FFI participated in Packet-Speech experiments performed in 1978-1979 in collaboration with among others MIT Lincoln Laboratories just outside Boston. The packet speech activity was part of the SATNET project. Lincoln Lab had developed a speech vocoder (voice coder and decoder), under contract with DARPA, providing a stream of $2.4 \mathrm{~kb} / \mathrm{s}$ digitized speech. The vocoder was interfaced with the PDP-11/45 at FFI, with a similar arrangement at MIT Lincoln Lab, and later at UCL. During the speech experiments, the PDP-11/45 acted then not as gateways, but as speech host. In addition, the PDP-11/45 contained a conference management program, also developed by Lincoln Lab, which handed over the "floor" in a FI-FO queue manner, to the parties indicating their wishes to talk.

Pål Spilling performed a set of measurements to determine the profile of packetspeech traffic. The programming of the PDP-11/45, performed in connection with the 
experiments, is a good example of resource sharing. The computer was located nextdoor to Spilling's office. The programming tools were located in a machine at Information Sciences Institute (ISI) in Los Angeles. Using a terminal in his office, connected to NORSAR-TIP, Spilling could log on to the computer in Los Angeles, write the necessary modifications to the control program, and have it compiled and uploaded across the network into the PDP-11/45 next door to Spilling's office. A socalled cross-network debugger (X-NET), also in the TOPS-20 machine, facilitated the downloading. This enabled Spilling to debug the modified control program loaded into the PDP-11/45 [8], before it was used for the experiments.

FFI participated in several packet-speech demonstrations. At one of the regular project meetings, held at UCL, Yngvar Lundh could not attend in person. $\mathrm{He}$ therefore made use of the conference facility and thereby could participate in the meeting from Norway. The quality of the speech when compressed to $2.4 \mathrm{~kb} / \mathrm{s}$ was noticeably impaired, but packet transmission through this early internet connection worked fine in real time.

In 1979-80, Pål Spilling stayed sixteen months with SRI International in Menlo Park California, working on the ARPA-funded Packet Radio Network (PRNET). There he made a proposal to improve the software architecture in the PR-nodes to have a better logical layering of the program structure [9]. He also performed extensive experiments on packet-speech performance with QoS-control [10], and suggested a "Busy Tone" method to overcome the "hidden terminal" problem in wireless communications [11].

\section{Competition between Alternatives}

Simultaneously with the growth of the ARPANET in the early 1970s, we saw the emergence of other competing communication concepts, like CYCLADE in France presented by Pouzin in 1973, the European Informatics Network (EIN) [12] presented in 1976, and the CCITT's Orange Books containing the X.25 standards published in 1977 [13]. In 1983, the International Standards activities presented the "Reference Model for Open Systems" and then in succession a set of layered communication protocols [14]. The dominating feature of X.25, and the ISO standards in general, was the virtual circuit principle, in contrast to the flexible connection-less datagram mode of operation in the ARPANET and later the internet. A virtual circuit in the packet switched arena is the equivalent of the end-to-end circuit establishment in the telephone network. The dominant part of the Norwegian research community, including NTA-R\&D was for a long time convinced that packet communications had to be based on virtual circuits.

The management at FFI and NTA-R\&D, and the Norwegian communication research community at large did not believe in the internet technology before the end of the 1980s and the beginning of the 1990s. In general, most communication experts believed that the TCP/IP suite of protocols eventually would be replaced by internationally agreed standards. Therefore, when we attempted to create interests for participation in the further development of this technology, the responses were negative. 


\section{International Communication Standards Activities}

The national authorities and the academic communities believed strongly in international standards. It was relatively easy to obtain funding for participation in standards activities. As seen from the outside, the standards work was less committing than the intense work that went on in the DARPA community. Standards were worked out on paper within a study period of four years, and when ready accepted more or less without practical experience. Later, when standards were to be implemented and tested out, deficiencies were surely detected and the standards had to be revised, and then re-proposed as standard in the next study period. In contrast the DARPA research went via specifications, implementations, testing, modifications, more testing and refinements, and when mature and stable enough, finally adopted as standard. This included also a set of support functions like "name to address mapping," "service type to service-access-point mapping," and "IP address to MAC address mapping," to make the combined protocol layers work efficiently and user friendly.

When the ISO communication standards came out in the middle and latter half of the 1980s, after a substantial work effort, a set of standards had been defined for each layer in the reference model. These standards included many options. Before the standards could be implemented, one had to make use of workshops to agree on the options to use in practice. This took quite a while. It is worth mentioning that the options agreed upon, made the ISO standards, for all practical purposes, functionally equivalent to the internet protocols.

Agreed international standards were not openly available. They had to be purchased for a certain fee. In contrast, all internet protocol specifications and related documentations were freely available.

\section{What Did Norway Benefit from Participating in the Development Project?}

In the middle of 1982, Spilling was invited to move over to Telenor-R\&D. He got the impression before he moved that they had interests in the internet technology. This turned out to be wrong. Only one of the research scientists there was interested in participating in the internet project. However, an advantage of being a member of the research staff at Telenor-R\&D gave Spilling the opportunity to establish a small Norwegian internet. It was operational from 1983-84 through 1986-87, interconnecting informatics departments at the universities in Oslo, Bergen and Trondheim, and NTA-R\&D. The network at NTA-R\&D was interconnected, via the Nordic satellite ground station at Tanum and SATNET, with ARPANET in the U.S. Due to his participation in the internet project, Spilling was able to obtain the Berkeley version of UNIX. This UNIX version had the whole internet protocol suite integrated in the system. This implied that influential Norwegian academic research people obtained experience with UNIX and could make use of internet services and communicate with colleagues in the U.S. This demonstrated that the internet communications worked well and provided a set of reliable, effective, and attractive services. 
UNINETT, the Norwegian academic communications network, was established in the first half of the 1980s. The goal was to interconnect the computer centers and the main research groups of the Norwegian universities. Due to political pressures, the network should be based on European supported connection-oriented standards.

In 1984-85, a major grant was given to the academic networks in the Nordic countries, with the goal to interconnect the national networks. Due to the very slow progress in the ISO standardization process, a multiprotocol transport service was developed and established, providing X.25, DECNET, IP, and possibly other alternatives. These interconnected networks, called NORDUNET, had its interconnection hub located in Stockholm. From there were leased lines to the Center for Mathematics and Computer Science (CWI) in Amsterdam, to CERN in Switzerland, and to the internet in USA (Princeton). This meant that all the academic networks in the Nordic countries got access to the internet. Gradually IP got a solid foothold, with the result that the other alternatives faded away. This was very fortunate when the SATNET project was terminated in 1989, because now all Norwegian IP-based traffic went via the hub in Stockholm.

The knowledge and experiences gained in participating in the DARPA projects led to the establishment of a computer communications research group and an early curriculum in computer communications at the Department of informatics at the Oslo University. Yngvar Lundh and Pål Spilling initiated this effort and gradually led to the establishment of similar activities at all universities in Norway.

\section{Discussions and Conclusions}

For a long time, there was a low interest in Europe, including Norway, regarding the internet technology. The opinion among communications research people in Norway was that the internet technology in a few years would be replaced by agreed international standards, like X.25 or ISO. For the Norwegian participation in the internet project, this resulted more or less in a one-man-show performed by Spilling. This of course greatly reduced the opportunity for a broader and more powerful contribution.

In December of 1973, half a year after NORSAR-TIP was installed, a Norwegian ARPANET committee was established to promote and coordinate possible Norwegian participations in DARPA activities. It consisted of members from the Research Council of Norway, and various research organizations. A condition for connecting to NORSAR-TIP, or making use of its terminal service, was that this should contribute to the furthering of the technology and be beneficial to both FFI and NDRE. The committee did not come up with any constructive proposals and dissolved itself in 1975.

When the internet technology, including its basic user services inherited from the ARPANET, was accepted as a standard for the American defense in 1981-82, it was about ten to fifteen years ahead of the marked. The network was mainly used by research people, and was not open to everyone. The first commercial web browser (Mosaic) was available in 1993. Approximately, at the same time the network was opened up for commercial use, resulting in an explosion in the desire to use the internet and its services. 
When the internet protocol (IP) was developed and standardized, it contained in its header two 32-bit addresses, one for the destination address and one for the source address. This was thought to be more than sufficient. However, it turned out to be far too small in order to cope with the globalization of the internet. Nobody at that time had the faintest idea about the immense use of the internet today, in the public domain, in the business domain, and in the private sector.

The internet technology gradually convinced the whole world that this technology was efficient, reliable, and easy to administer, in contrast to the CCITT's X.25 concept and the ISO standards. American companies dominated the production of computers and their software, and they were delivered with the internet protocols integrated in their operating systems. They were unwilling to implement, say the ISO standards, unless customers (European companies) paid the costs. Moreover, nobody did that. This meant that very few people, globally speaking, were exposed to other alternatives.

There was a general antipathy in Europe, including Norway, regarding defense matters, and especially everything that was connected with the U.S. defense. This contributed to the low interest, in Norway and in Europe as a whole, for the internet technology.

The Nordic countries have the highest percentage of internet users in the world. This is in part due to the early exposure to this technology, first in the academic world and later in the public sector. NTA (Telenor) was among the first telecom operators in Europe, around 1994-95, to be convinced to offer internet access to customers. This is certainly due to the close exposure to this technology over a long period at NTA-R\&D.

\section{References}

1. Roberts, L., Wessler, B.D.: Computer Network Development to Achieve Resource Sharing. In: Spring AFIPS Conf. (1970)

2. Cerf, V., Kahn, R.E.: A Protocol for Packet Network Interconnection. IEEE Trans. Comm. Tech. (1974)

3. Kahn, R.E., Gronemeyer, S., Burchfiel, J., Kunzelman, R.: Advances in Packet Radio Technology. Proc. IEEE (November 1978)

4. Jacobs, I., Hoversten, E.: General Purpose Packet Satellite Network. In: Proc. IEEE Spec. Issue on Packet Communications Network (November 1978)

5. Davies, D.W.: Communication Networks to Serve Rapid Response Computers. In: IFIP Congress (August 1968)

6. Spilling, P., Lundh, Y.: Features of the Internet History, The Norwegian Contribution to the Development. Telektronikk 3, 113 (2004)

7. Spilling, P., Lundh, Y., Aagesen, F.A.: Final Report on the Packet Satellite Program. Internal Report E-290, FFI-E (1978)

8. Spilling, P., McElwain, Forgie J.W.: Packet Speech and Network Performance. Internal Report E-295, FFI-E (1979)

9. Spilling, P.: Low-Cost Packet Radio Protocol Architecture and Functions - Preliminary Requirements. Technical Note 1080-150-1, SRI International (October 1980)

10. Spilling, P., Craighill, E.: Digital Voice Communications in the Packet Radio Network. In: ICC 1980, Seattle (1980) 
11. Spilling, P., Tobagi, F.: Activity Signaling and Improved Acknowledgements in Packet Radio Systems. Packet Radio Technical Note 283, SRI International (1980)

12. Porcet, F., Repton, C.S.: The EIN Communication Subnet Principles and Practice. In: Proc. of ICCC, Toronto (1976)

13. Series X Recommendations. The Orange Book, ITU, Geneva (1977)

14. Basic Reference Model. ISO 7498-1 (1983)

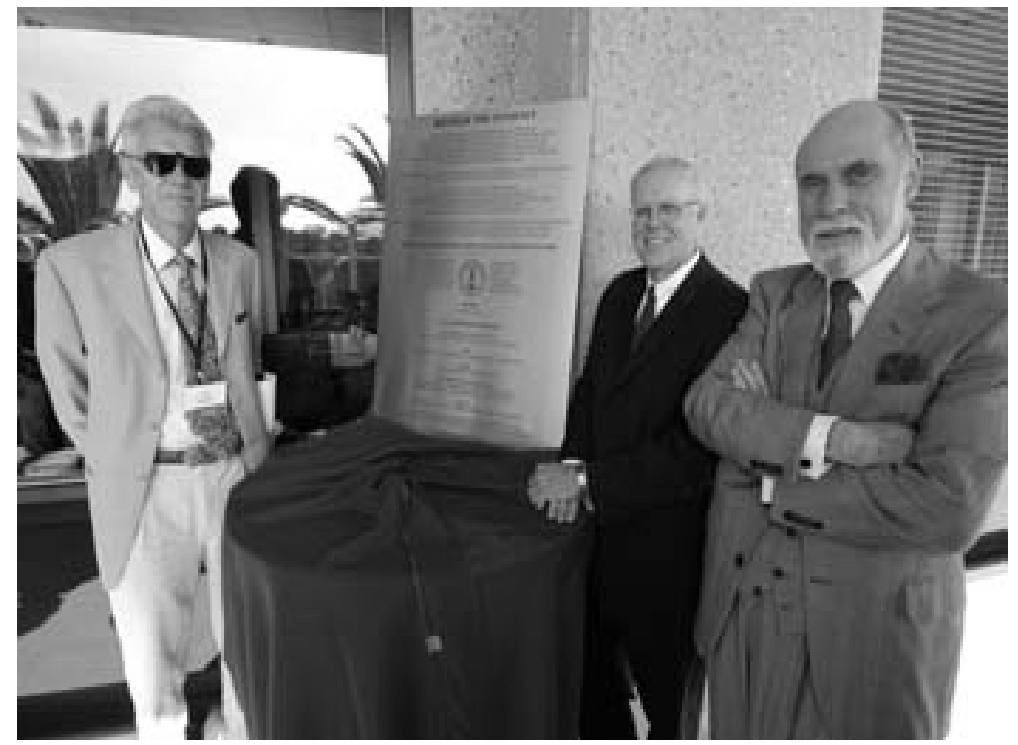

From the ceremony at Stanford University in 2005, celebrating thirty years since the start of the internet development. We see from left to right: Pål Spilling, President John Hennessy of Stanford University, and Vinton Cerf. There are thirty-two names on the bronze plaque, among which Dag Belsnes, Yngvar Lundh, and Pål Spilling are from Norway. 